\title{
Bath Ankylosing Spondylitis Disease Activity Index is Associated With the Quality of Sleep in Ankylosing Spondylitis Patients
}

\author{
Byung Wook Song, M.D. ${ }^{1}{ }^{*}$, Hye-Jin Jeong, M.D. ${ }^{*}$, , Bo Young Kim, M.D. ${ }^{2}$, Yong Won Cho, M.D., Ph.D. ${ }^{3}$, \\ Chang-Nam Son, M.D., Ph.D. ${ }^{1}$, Sung-Soo Kim, M.D., Ph.D. ${ }^{2}$, Sang-Hyon Kim, M.D., Ph.D. ${ }^{1}$ \\ ${ }^{1}$ Division of Rheumatology, Department of Internal Medicine, Keimyung University School of Medicine, Daegu, ${ }^{2}$ Division of Rheumatology, \\ Department of Internal Medicine, Gangneung Asan Hospital, University of Ulsan College of Medicine, Gangneung, ${ }^{3}$ Department of Neurology, \\ Keimyung University School of Medicine, Daegu, Korea
}

\begin{abstract}
Objective. High disease activity of ankylosing spondylitis (AS) is associated with poor sleep quality. The purpose of this study was to identify which of the representative tools for evaluating the disease activity of AS best reflect the quality of sleep. Methods. A total of 107 AS patients were enrolled in the study and the sleep quality was assessed using the Pittsburgh Sleep Quality Index (PSQI). Age, sex, concomitant medication, erythrocyte sedimentation rate (ESR), serum C-reactive protein (CRP) level, Beck Depression Inventory second edition (BDI-II), Bath ankylosing spondylitis disease activity index (BASDAI), ankylosing spondylitis disease activity score (ASDAS)-ESR, ASDAS-CRP, pain visual analog scale, Insomnia Severity Index (ISI), and Epworth Sleepiness Scale (ESS) were analyzed as covariates. Results. Overall, 65\% (70/107) of subjects reported poor sleep quality (PSQI > 5). There was a positive correlation between the sleep quality and disease activity as measured by the BASDAI, ASDAS-ESR, and ASDAS-CRP. In addition, the BASDAI demonstrated good correlations with ISI, ESS, and BDI-II, respectively. However, only BASDAI showed reliable correlation with PSQI among the disease activity parameters of AS (adjusted odd ratio $5.36, p=0.023)$. Conclusion. BASDAI is the most reliable parameter of disease activity associated with the sleep quality in patients with AS. (J Rheum Dis 2021;28:143-149)
\end{abstract}

Key Words. Ankylosing spondylitis, Bath ankylosing spondylitis disease activity index, Sleep quality

\section{INTRODUCTION}

Ankylosing spondylitis (AS) is a seronegative spondyloarthropathy with chronic inflammation around the spine and pelvis as the main symptom [1]. The disease onset is at a relatively young age, and the duration of the disease is relatively long. Hence, the patient's quality of life is greatly affected by the progression of the disease [1].
It is well known that the quality of sleep is poor among Koreans with rheumatic diseases such as rheumatoid arthritis, AS, and Behçet's disease [2-4]. Recent meta-analyses demonstrated that the quality of sleep in patients with AS is reduced by approximately $35 \% \sim 90 \%$ compared to that in healthy individuals $[5,6]$ and that higher disease activity has more impact on the sleep quality. In particular, back pain and stiffness are associated with

Received : April 13, 2021, Revised : April 19, 2021, Accepted : April 22, 2021

Corresponding to : Sang-Hyon Kim (ib http://orcid.org/0000-0002-8030-7939

Division of Rheumatology, Department of Internal Medicine, Keimyung University School of Medicine, 1035 Dalgubeol-daero, Dalseo-gu, Daegu 42601, Korea. E-mail : mdkim9111@hanmail.net

Sung-Soo Kim (1Dhttp://orcid.org/0000-0002-8556-343X

Division of Rheumatology, Department of Internal Medicine, Gangneung Asan Hospital, University of Ulsan College of Medicine, 38 Bangdong-gil, Sacheon-myeon, Gangneung 25440, Korea. E-mail : drkiss@ulsan.ac.kr

Chang-Nam Son (iD http://orcid.org/0000-0002-1722-2190

Division of Rheumatology, Department of Internal Medicine, Keimyung University School of Medicine, 1035 Dalgubeol-daero,

Dalseo-gu, Daegu 42601, Korea. E-mail : cnson@kmu.ac.kr

*These authors contributed equally to this work. 
sleep disturbances. Besides disease activity, pain, decline in body functions, and mood have been found to affect the sleep quality [5].

The use of biologic agents such as tumor necrosis factor-alpha (TNF- $\alpha$ ) inhibitors has dramatically improved the treatment of AS. Biologic agents not only improve the quality of life but have also been recently found to prevent radiological progression [7]. It has not been established yet whether a biologic agent can improve the quality of sleep in patients with AS. However, it is crucial to find an indicator that reflects the disease activity and sleep quality accurately, and this can be used as an important assessment tool for optimal treatment of patients.

Tools for assessing the disease activity in AS patients include the Bath ankylosing spondylitis disease activity index (BASDAI), ankylosing spondylitis disease activity score-erythrocyte sedimentation rate (ASDAS-ESR), and ankylosing spondylitis disease activity score-C-reactive protein (ASDAS-CRP) [8]. The use of each of these indicators is associated with certain advantages and disadvantages. Therefore, we aimed to determine which of these indicators used for assessing the disease activity of AS best reflects the sleep quality.

\section{MATERIALS AND METHODS}

\section{Study population}

A total of 107 patients with AS who visited two tertiary rheumatology clinics in Korea were enrolled from September 2019 to October 2020.

Inclusion criteria for the patients were: age 19 years or older and meeting the 1984 modified New York criteria [9]. Exclusion criteria were: being uneducated and unable to answer the questionnaire; having low mental aptitude; having a history of medical diseases such as hypertension, diabetes mellitus, fibromyalgia, malignant tumor, or infection, which can affect the quality of sleep; or being suspected to be drug addicts (including sleeping pills or alcohol).

\section{Ethics statement}

This study was approved by the Institutional Review Board (IRB) at the university hospital where the study was conducted (IRB numbers: KUDH 2019-07-032 and GNAH 2019-10-014) and was conducted in accordance with the Declaration of Helsinki. Written informed consent was obtained from all study participants.

\section{Assessment of clinical variables}

Along with a questionnaire, face-to-face interviews were conducted with the patients with AS. Age, sex, body mass index (BMI), visual analogue scale (VAS) score for pain, and other associated disease history were recorded and analyzed. Sleep quality, depression, and disease activity of AS were also scrutinized. From the medical records, additional information was collected, such as blood test results for C-reactive protein (CRP), erythrocyte sedimentation rate (ESR), and human leukocyte antigen (HLA) B27 positivity; disease duration; and medical history associated with disease activity of AS.

\section{Assessment of sleep quality}

Quality of sleep was evaluated using the Korean version of the Pittsburgh Sleep Quality Index (PSQI) [10]. PSQI consists of a questionnaire regarding sleep habits over the past one month and is a useful test to evaluate the sleep quality. It contains seven subsections: subjective sleep quality, sleep latency, sleep duration, habitual sleep efficiency, sleep disturbances, use of sleeping medication, and daytime dysfunction. Each of these components has a range of $0 \sim 3$ points, with the total adding up to $0 \sim 21$ points. Higher scores indicate poor sleep quality; when the total score was over 5 , patients were classified into the poor sleeper group.

\section{Assessment of insomnia}

Severity of insomnia was evaluated using the Korean version of Insomnia Severity Index (ISI) [11]. ISI consists of a questionnaire in which the respondents are asked to rate, on a range of $0 \sim 4$ points, the nature and symptoms of their sleep problems. It contains five subsections: the severity of symptoms, the respondent's satisfaction with his or her sleep patterns, the degree to which insomnia interferes with daily functioning, how noticeable the respondent feels his or her insomnia is to others, and the overall level of distress created by the sleep problem. By adding the scores of each component, a total score from 0 to 28 points is achieved. A total score of $0 \sim 7$ indicates no clinically significant insomnia, $8 \sim 14$ means subthreshold insomnia, $15 \sim 21$ is moderate severity clinical insomnia, and 22 28 indicates severe clinical insomnia [11].

\section{Assessment of daytime sleepiness}

Daytime sleepiness was measured using the Korean version of the Epworth Sleepiness Scale (ESS) [12]. ESS con- 
sists of a questionnaire in which the respondents are asked to rate, on a range of $0 \sim 3$ points, their usual chances of dozing off or falling asleep during eight different activities during the daytime. Higher ESS scores indicate an average person's higher sleep propensity in daily life; when the total score is more than 11 , it is defined as excess daytime sleepiness [12].

\section{Assessment of depression}

The Korean version of the Beck Depression Inventorysecond edition (BDI-II) was used to evaluate the degree of depression [13].

BDI-II consists of 21 multiple-choice self-report inventories, with points ranging from $0 \sim 3$. By adding the scores of each component, a total score ranging from 0 to 63 points is achieved. Higher scores indicate severe depression, and 20 points has been defined as the cut-off point for depression [13].

\section{Assessment of ankylosing spondylitis disease activity} The BASDAI [14] as well as ASDAS-CRP and ASDASESR [15] were evaluated as indicators of AS disease activity. To assess the disease activity, we used the Korean versions of the BASDAI, ASDAS-CRP, and ASDAS-ESR.

The BASDAI questionnaire contains six questions regarding subjective symptoms during the week prior to answering the questions. Each question is scored on a scale of 0 to 10. According to the Assessment of Spondyloarthritis International Society criteria, a BASDAI score $\geq 4$ was considered as high disease activity, while a score $<2$ was considered as remission [16].

The ASDAS-CRP and ASDAS-ESR take into an account both patient-perceived symptoms as well as acute-phase reactants. These composite indices take into account three questions of the BASDAI, patient's global assessment, as well as the CRP or ESR values. Scores range from 0 (no disease activity) to infinity (being determined by the level of CRP or ESR). The cutoffs between the disease activity states are: inactive disease $\leq 1.3$, moderate: $1.3 \sim$ 2.0, high: $2.1 \sim 3.5$, and very high $\geq 3.5$ [17].

\section{Statistical analysis}

The statistical program SPSS for Windows, version 26.0 (IBM Co., Armonk, NY, USA), was used for data processing and statistical analysis. The continuous data were analyzed as means and standard deviations, while categorical data were assessed as frequencies and percentages. The independent $t$ test was used to compare the means between the groups, while the chi-square test was used to compare the proportions. The correlation between variables was calculated with Pearson's correlation coefficient. The crude or adjusted odds ratios (ORs) were estimated using simple or multiple logistic regression analysis. All

Table 1. The demographic characteristics of the study population

\begin{tabular}{|c|c|}
\hline Variable & $\begin{array}{l}\text { Total population } \\
\qquad(\mathrm{n}=107)\end{array}$ \\
\hline Sex, male & $88(82.2)$ \\
\hline Age (yr) & $35.81 \pm 11.78$ \\
\hline BMI $\left(\mathrm{kg} / \mathrm{m}^{2}\right)$ & $24.66 \pm 4.09$ \\
\hline $\mathrm{ESR}(\mathrm{mm} / \mathrm{hr})$ & $17.94 \pm 17.83$ \\
\hline $\mathrm{CRP}(\mathrm{mg} / \mathrm{dL})$ & $0.32 \pm 0.77$ \\
\hline HLA B27, positive & $92(86.0)$ \\
\hline Disease duration (mo) & $71.92 \pm 71.73$ \\
\hline TNF- $\alpha$ inhibitor use & $76(71.0)$ \\
\hline Pain (VAS) $(\mathrm{cm})$ & $2.48 \pm 1.84$ \\
\hline Total BASDAI & $3.10 \pm 1.68$ \\
\hline Fatigue & $5.28 \pm 2.37$ \\
\hline Total back pain & $3.44 \pm 2.37$ \\
\hline Peripheral pain/swelling & $2.30 \pm 2.21$ \\
\hline Enthesitis & $1.55 \pm 2.10$ \\
\hline Level of morning stiffness & $3.29 \pm 2.52$ \\
\hline Duration of morning stiffness & $2.55 \pm 2.36$ \\
\hline \multicolumn{2}{|l|}{ ASDAS } \\
\hline Total back pain & $3.44 \pm 2.37$ \\
\hline Duration of morning stiffness & $2.55 \pm 2.36$ \\
\hline Patient global assessment & $3.10 \pm 1.94$ \\
\hline Peripheral pain/swelling & $2.30 \pm 2.21$ \\
\hline Total ASDAS-ESR & $2.11 \pm 0.88$ \\
\hline Total ASDAS-CRP & $1.83 \pm 0.74$ \\
\hline ISI & $8.42 \pm 5.49$ \\
\hline ESS & $6.50 \pm 3.88$ \\
\hline PSQI & $7.00 \pm 3.25$ \\
\hline Subjective sleep quality & $1.51 \pm 0.65$ \\
\hline Sleep latency & $1.50 \pm 1.05$ \\
\hline Sleep duration & $0.91 \pm 0.96$ \\
\hline Habitual sleep efficiency & $0.33 \pm 0.74$ \\
\hline Sleep disturbance & $1.17 \pm 0.50$ \\
\hline Use of sleeping medicine & $0.17 \pm 0.61$ \\
\hline Daytime dysfunction & $1.43 \pm 0.93$ \\
\hline BDI-II & $11.63 \pm 8.05$ \\
\hline
\end{tabular}

All values are numbers (\%) or means \pm standard deviations. ASDAS: ankylosing spondylitis disease activity score, BASDAl: bath ankylosing spondylitis disease activity index, BDI: Beck Depression Inventory, BMI: body mass index, CRP: C-reactive protein, ESR: erythrocyte sedimentation rate, ESS: Epworth Sleepiness Scale, HLA B27: Human Leucocyte Antigen B27, ISI: insomnia severity index, PSQI: Pittsburgh Sleep Quality Index, TNF- $\alpha$ : tumor necrosis factor-alpha, VAS: Visual Analogue Scale. 
the results with a p-value less than 0.05 were considered as significant.

\section{RESULTS}

\section{Demographic and clinical characteristics of the patients with ankylosing spondylitis}

A total of 107 patients were enrolled in this study and completed the questionnaire. Among them, 88 patients were men $(82.2 \%)$, with mean age of $35.81 \pm 11.78$ years and BMI of $24.66 \pm 4.09 \mathrm{~kg} / \mathrm{m}^{2}$. The average ESR was $17.94 \pm 17.83 \mathrm{~mm} / \mathrm{hr}$, and the CRP was $0.32 \pm 0.77 \mathrm{mg} / \mathrm{dL}$. There were 92 (86\%) patients with positive HLA B27, and the mean duration of the disease was $71.92 \pm 71.73$ months. The mean VAS score was $2.48 \pm 1.84 \mathrm{~cm}$. The mean BASDAI was 3.10 \pm 1.68 , ASDAS-ESR was $2.11 \pm$ 0.88 , and ASDAS-CRP was $1.83 \pm 0.74$. The mean ISI was 8.42 \pm 5.49 , mean ESS was 6.50 \pm 3.88 , mean BDI-II was $11.63 \pm 8.05$, and mean PSQI was $7.00 \pm 3.25$ (Table 1).

\section{Comparison between the good sleeper and poor sleeper groups}

Out of the 107 patients in total, 37 (34.6\%) were good sleepers and $70(65.4 \%)$ were poor sleepers. There was no significant difference between men and women in both groups. Furthermore, there were no significant differences in the BMI, ESR, CRP, HLA status, duration of disease, and the use of TNF- $\alpha$ inhibitor. However, there was a significant difference in the mean age between the two groups. The mean ages of good sleepers and poor sleepers were $40.38 \pm 13.46$ and $33.40 \pm 10.08$ years, respectively $(\mathrm{p}<0.05)$. The VAS, BDI-II, BASDAI, ASDASESR, and ASDAS-CRP scores also showed significant differences between the two groups $(p<0.05)$ (Table 2$)$.

\section{Correlation between sleep quality parameters and the disease activity parameters}

There were positive correlations between the tools for assessing the sleep quality (PSQI, ISI, ESS) and disease activity (BASDAI, ASDAS-ESR, ASDAS-CRP). The correlation of PSQI with BASDAI was the highest at 0.469, followed by ASDAS-CRP at 0.373, and ASDAS-ESR at 0.290 . In addition, the ISI and ESS demonstrated correla-

Table 3. Correlation between the sleep quality indices and disease activity indices of ankylosing spondylitis

\begin{tabular}{lccc}
\hline \hline Parameters & PSQI & ISI & ESS \\
\hline BASDAI & $0.469^{* *}$ & $0.523^{* *}$ & $0.525^{* *}$ \\
ASDAS-ESR & $0.290^{* *}$ & $0.347^{* *}$ & $0.366^{* *}$ \\
ASDAS-CRP & $0.373^{* *}$ & $0.429^{* *}$ & $0.405^{* *}$ \\
\hline
\end{tabular}

ASDAS: ankylosing spondylitis disease activity score, BASDAI: bath ankylosing spondylitis disease activity index, CRP: C-reactive protein, ESR: erythrocyte sedimentation rate, ESS: Epworth Sleepiness Scale, ISI: insomnia severity index, PSQI: Pittsburgh Sleep Quality Index. ${ }^{*} \mathrm{p}<0.01$.

Table 2. Comparison between the good sleeper and poor sleeper groups

\begin{tabular}{lccc}
\hline \multicolumn{1}{c}{ Variable } & Good sleeper (PSQI $\leq 5)$ & Poor sleeper (PSQI $>5)$ & p-value \\
\hline Total & $37(34.6)$ & $70(65.4)$ & 0.970 \\
Sex, male & $31(83.8)$ & $57(81.4)$ & 0.008 \\
Age $(\mathrm{yr})$ & $40.38 \pm 13.46$ & $33.40 \pm 10.08$ & 0.850 \\
BMI $\left(\mathrm{kg} / \mathrm{m}^{2}\right)$ & $24.76 \pm 3.39$ & $24.60 \pm 4.43$ & 0.254 \\
ESR $(\mathrm{mm} / \mathrm{hr})$ & $15.49 \pm 13.96$ & $19.24 \pm 19.54$ & 0.441 \\
CRP $(\mathrm{mg} / \mathrm{dL})$ & $0.25 \pm 0.46$ & $0.35 \pm 0.89$ & 0.384 \\
HLA B27, positive & $30(81.1)$ & $62(88.6)$ & 0.107 \\
Disease duration (mo) & $87.30 \pm 81.15$ & $63.79 \pm 65.39$ & 0.922 \\
TNF- $\alpha$ inhibitor use & $27(73.0)$ & $49(70.0)$ & 0.016 \\
Pain (VAS) (cm) & $1.89 \pm 1.81$ & $2.79 \pm 1.80$ & $<0.001$ \\
BDI-II & $7.16 \pm 5.86$ & $13.99 \pm 8.08$ & $<0.001$ \\
BASDAI & $2.09 \pm 1.34$ & $3.63 \pm 1.60$ & 0.002 \\
ASDAS-ESR & $1.76 \pm 0.72$ & $2.30 \pm 0.91$ & 0.002 \\
ASDAS-CRP & $1.54 \pm 0.62$ & $2.00 \pm 0.75$ & \\
\hline
\end{tabular}

All values are numbers (\%) or means \pm standard deviations. ASDAS: ankylosing spondylitis disease activity score, BASDAI: bath ankylosing spondylitis disease activity index, BDI: Beck Depression Inventory, BMI: body mass index, CRP: C-reactive protein, ESR: erythrocyte sedimentation rate, HLA B27: Human Leucocyte Antigen B27, PSQI: Pittsburgh Sleep Quality Index, TNF- $\alpha$ : tumor necrosis factor-alpha, VAS: Visual Analogue Scale. 
tions with the BASDAI, ASDAS-ESR, and ASDAS-CRP scores (Table 3). Additionally, we analyzed the correlation between the detailed items constituting the PSQI and the detailed items of the disease activity evaluation indicators of AS (Supplementary Table 1). Among the detailed items of the disease activity evaluation indicators, fatigue was the most correlated with the total PSQI score $(r=0.465$, $\mathrm{p}<0.01)$.

\section{Relationship between the sleep quality and disease activity}

On comparing the odds ratios between the groups with well-controlled AS and poorly-controlled AS and between the poor sleepers and good sleepers, the crude OR was the highest for BASDAI at 4.27 (Table 4), followed by ASDAS-CRP at 2.56, and ASDAS-ESR at $2.46(\mathrm{p}<0.05)$. After adjustment for age, VAS, and BDI-II, which showed significant differences between the poor sleeper and good sleeper groups as shown in Table 2, the adjusted odds ratio was the highest for BASDAI at 5.36, followed by ASDAS-CRP at 2.74, and ASDAS-ESR at 2.61, but was significant only for BASDAI $(\mathrm{p}=0.023)$.

\section{DISCUSSION}

In the past, healthcare only focused on the treatment of acute diseases such as infections. However, with the development of medicine and emergence of an aging society, the focus is moving towards the treatment and management of chronic diseases. Consequently, modern medicine pays considerable attention to the improvement of the quality of life, sleep disorders, depression, and stress, which can be affected by diseases as well as the termination or alleviation of physical symptoms related to the diseases. Among them, sleep affects many functions such as memory, emotion control, recovery of body functions, metabolic regulation, immune function, and inflammation control $[18,19]$. Rheumatic diseases are mostly chronic inflammatory autoimmune diseases, and ensuring proper sleep can affect the control of the disease activity. An important goal of treatment of AS, which is a chronic inflammatory autoimmune disease occurring at a relatively young age, is the improvement of the sleep disorder or psychological factors in addition to simple pain relief since the duration of the disease is long. Consequently, we studied the relationship between disease activity and sleep quality in patients with AS.

Several previous studies, including a systematic literature review and meta-analysis, have shown that the factors influencing the sleep disorder in AS include demographic factors such as age, sex, and education; disease-related factors such as disease activity, fatigue, degree of body function, and pain; and psychological factors such as depression, anxiety, and quality of life. The strength of the correlations appears to be slightly different in each study $[5,6,20-23]$. In a meta-analysis of six studies that analyzed patients with AS and healthy controls using the PSQI as an index for the evaluation of sleep quality, the BASDAI score did not show a significant correlation with the total PSQI score [6]. Nevertheless, many studies have reported that the BASDAI, an evaluation index of disease activity, has a high correlation with sleep quality [5]. However, there has been no international study that has used ASDAS as an evaluation index for disease activity. Similar to previous studies that reported that high disease activity was correlated with poor sleep quality [3,5,6,21-24], our study also revealed that the group with low sleep quality (poor sleepers) demon-

Table 4. Relationship between the sleep quality and disease activity

\begin{tabular}{|c|c|c|c|c|c|}
\hline \multirow{2}{*}{\multicolumn{2}{|c|}{ Parameters }} & \multicolumn{2}{|l|}{ Crude } & \multicolumn{2}{|c|}{ Adjusted* } \\
\hline & & \multirow{2}{*}{$\frac{\mathrm{OR}(95 \% \mathrm{Cl})}{4.27(1.48 \sim 12.28)}$} & \multirow{2}{*}{$\frac{p \text {-value }}{0.007}$} & \multirow{2}{*}{$\frac{\mathrm{OR}(95 \% \mathrm{Cl})}{5.36(1.26 \sim 22.69)}$} & \multirow{2}{*}{$\frac{p \text {-value }}{0.023}$} \\
\hline BASDAI & $<4$ & & & & \\
\hline & $\geq 4$ & 1.00 & & 1.00 & \\
\hline \multirow[t]{2}{*}{ ASDAS-ESR } & $<2.1$ & $2.46(1.08 \sim 5.61)$ & 0.032 & $2.61(0.92 \sim 7.42)$ & 0.073 \\
\hline & $\geq 2.1$ & 1.00 & & 1.00 & \\
\hline \multirow[t]{2}{*}{ ASDAS-CRP } & $<2.1$ & $2.56(1.03 \sim 6.41)$ & 0.044 & $2.74(0.87 \sim 8.67)$ & 0.086 \\
\hline & $\geq 2.1$ & 1.00 & & 1.00 & \\
\hline
\end{tabular}

ASDAS: ankylosing spondylitis disease activity score, BASDAI: bath ankylosing spondylitis disease activity index, CRP: C-reactive protein, ESR: erythrocyte sedimentation rate, OR: odds ratio, $\mathrm{Cl}$ : confidence intervals, VAS: Visual Analogue Scale, BDI-II: Beck Depression Inventory-II. *Adjusted for age, VAS, BDI-II. 
strated higher disease activity, pain, and depression indices.

Between BASDAI and ASDAS, which are used as evaluation indices for disease activity in clinical practice, BASDAI showed a higher association with sleep quality. The possible reason that the BASDAI and ASDAS showed this difference, although they are disease activity indices with a high correlation between them, appears to be due to the difference in the items comprising each index. Among the items included in the BASDAI, fatigue, local tenderness, and level of morning stiffness are different from the ASDAS. The items included in the ASDAS but not in the BASDAI are patient global assessment and objective inflammatory markers such as ESR/CRP $[14,15]$. As can be seen from Supplementary Table 1, which shows the result of the correlation analysis between the detailed items of each of the disease activity indices and the PSQI, fatigue exhibited the highest correlation with the total PSQI, whereas local tenderness, level of morning stiffness, patient global assessment, and ESR/CRP did not show high correlations. This suggests that the BASDAI index has a greater association with the sleep quality compared to the ASDAS since fatigue affects sleep quality the most, and the BASDAI includes fatigue. This is a valid conjecture considering the result of previous studies: the worse the fatigue, the worse is the sleep quality in patients with AS [25-28].

The limitations of our study are as follows: First, we did not perform radiological evaluation of the joint deformities that may interfere with sleep, such as spinal deformities. Second, we did not investigate objective indicators related to sleep, such as polysomnography and sleep-related cytokines. Third, we did not follow up a large number of patients for a sufficient period of time to observe whether a decrease in the disease activity indicates an improvement in the sleep quality or whether a biological agent leads not only to a decrease in the disease activity but also an improvement in the sleep quality.

\section{CONCLUSION}

This study found that high disease activity in AS was associated with a decrease in the sleep quality and that the BASDAI reflects this the best among the disease activity indices. We can expect improvement in the sleep quality as well as pain through active control of the disease activity in patients with AS.

\section{ACKNOWLEDGMENTS}

This work was supported by the National Research Foundation of Korea (NRF) Grant funded by the Korean Government (MSIP) (grant No. NRF-2018M3C1B7020722 and NFR-2017R1C1B5017278) and the research fund of the Rheumatology Research Foundation (RRF-2017-04).

\section{CONFLIC OF INTEREST}

No potential conflict of interest relevant to this article was reported.

\section{AUTHOR CONTRIBUTIONS}

Conceptualization: B.W.S., H.J.J., S.S.K., S.H.K., and C.N.S. Data curation: all authors. Formal analysis: B.W.S., H.J.J., S.S.K., B.Y.K., Y.W.C., S.H.K., and C.N.S. Funding acquisition: S.H.K., H.J.J., and C.N.S. Investigation: all authors. Methodology: Y.W.C., S.H.K., and C.N.S. Project administration: B.W.S., S.S.K., S.H.K., and C.N.S. Resources: all authors. Supervision: S.H.K., S.S.K., and C.N.S. Visualization: B.W.S., S.H.K., and C.N.S. Writing original draft: B.W.S., H.J.J., S.S.K., S.H.K., and C.N.S.

Writing - review and editing: all authors.

\section{SUPPLEMENTARY DATA}

Supplementary data can be found with this article online at https://doi.org/10.4078/jrd.2021.28.3.143.

\section{REFERENCES}

1. Sieper J, Rudwaleit M, Khan MA, Braun J. Concepts and epidemiology of spondyloarthritis. Best Pract Res Clin Rheumatol 2006;20:401-17.

2. Son CN, Choi G, Lee SY, Lee JM, Lee TH, Jeong HJ, et al. Sleep quality in rheumatoid arthritis, and its association with disease activity in a Korean population. Korean J Intern Med 2015;30:384-90.

3. Jeong HJ, Lee TH, Lee JM, Choi G, Son CN, Kim JM, et al. Sleep disturbances in Korean patients with ankylosing spondylitis are associated with increased disease activity. J Rheum Dis 2014;21:241-7.

4. Lee J, Kim SS, Jeong HJ, Son CN, Kim JM, Cho YW, et al. Association of sleep quality in Behcet disease with disease activity, depression, and quality of life in Korean population. Korean J Intern Med 2017;32:352-9.

5. Leverment S, Clarke E, Wadeley A, Sengupta R. Prevalence and factors associated with disturbed sleep in patients with ankylosing spondylitis and non-radiographic axial spondy- 
loarthritis: a systematic review. Rheumatol Int 2017;37: 257-71.

6. Li Z, Fu T, Wang Y, Dong C, Shao X, Li L, et al. Sleep disturbances in ankylosing spondylitis: a systematic review and meta-analysis. Psychol Health Med 2019;24:911-24.

7. Koo BS, Oh JS, Park SY, Shin JH, Ahn GY, Lee S, et al. Tumour necrosis factor inhibitors slow radiographic progression in patients with ankylosing spondylitis: 18-year real-world evidence. Ann Rheum Dis 2020;79:1327-32.

8. Kiltz U, Braun J. Assessments of functioning in patients with axial spondyloarthritis. J Rheum Dis 2020;27:22-9.

9. van der Linden S, Valkenburg HA, Cats A. Evaluation of diagnostic criteria for ankylosing spondylitis. A proposal for modification of the New York criteria. Arthritis Rheum 1984;27:361-8.

10. Sohn SI, Kim DH, Lee MY, Cho YW. The reliability and validity of the Korean version of the Pittsburgh Sleep Quality Index. Sleep Breath 2012;16:803-12.

11. Cho YW, Song ML, Morin CM. Validation of a Korean version of the insomnia severity index. J Clin Neurol 2014;10: 210-5.

12. Cho YW, Lee JH, Son HK, Lee SH, Shin C, Johns MW. The reliability and validity of the Korean version of the Epworth sleepiness scale. Sleep Breath 2011;15:377-84.

13. Lim SY, Lee EJ, Jeong SW, Kim HC, Jeong CH, Jeon TY, et al. The validation study of Beck Depression Scale 2 in Korean version. Anxiety Mood 2011;7:48-53.

14. Garrett S, Jenkinson T, Kennedy LG, Whitelock H, Gaisford $\mathrm{P}$, Calin A. A new approach to defining disease status in ankylosing spondylitis: the Bath Ankylosing Spondylitis Disease Activity Index. J Rheumatol 1994;21:2286-91.

15. Lukas C, Landewé R, Sieper J, Dougados M, Davis J, Braun $\mathrm{J}$, et al. Development of an ASAS-endorsed disease activity score (ASDAS) in patients with ankylosing spondylitis. Ann Rheum Dis 2009;68:18-24.

16. Cohen JD, Cunin P, Farrenq V, Oniankitan O, Carton L, Chevalier X, et al. Estimation of the Bath Ankylosing Spondylitis Disease Activity Index cutoff for perceived symptom relief in patients with spondyloarthropathies. J Rheumatol 2006;33:79-81.
17. Machado P, Landewé R, Lie E, Kvien TK, Braun J, Baker D, et al. Ankylosing Spondylitis Disease Activity Score (ASDAS): defining cut-off values for disease activity states and improvement scores. Ann Rheum Dis 2011;70:47-53.

18. Assefa SZ, Diaz-Abad M, Wickwire EM, Scharf SM. The functions of sleep. AIMS Neurosci 2015;2:155-71.

19. Zielinski MR, McKenna JT, McCarley RW. Functions and mechanisms of sleep. AIMS Neurosci 2016;3:67-104.

20. Da Costa D, Zummer M, Fitzcharles MA. Determinants of sleep problems in patients with spondyloarthropathy. Musculoskeletal Care 2009;7:143-61.

21. Nie A, Wang C, Song Y, Xie X, Yang H, Chen H. Prevalence and factors associated with disturbed sleep in outpatients with ankylosing spondylitis. Clin Rheumatol 2018;37: 2161-8.

22. Wadeley A, Clarke E, Leverment S, Sengupta R. Sleep in ankylosing spondylitis and non-radiographic axial spondyloarthritis: associations with disease activity, gender and mood. Clin Rheumatol 2018;37:1045-52.

23. Urkmez B, Keskin Y. Relationship between sleep quality and physical activity level in patients with ankylosing spondylitis. Mod Rheumatol 2020;30:1053-9.

24. Batmaz İ, Sarıyıldız MA, Dilek B, Bez Y, Karakoç M, Çevik R. Sleep quality and associated factors in ankylosing spondylitis: relationship with disease parameters, psychological status and quality of life. Rheumatol Int 2013;33:1039-45.

25. Missaoui B, Revel M. Fatigue in ankylosing spondylitis. Ann Readapt Med Phys 2006;49:305-8.

26. Günaydin R, Göksel Karatepe A, Ceşmeli N, Kaya T. Fatigue in patients with ankylosing spondylitis: relationships with disease-specific variables, depression, and sleep disturbance. Clin Rheumatol 2009;28:1045-51.

27. Aissaoui N, Rostom S, Hakkou J, Berrada Ghziouel K, Bahiri $\mathrm{R}$, Abouqal R, et al. Fatigue in patients with ankylosing spondylitis: prevalence and relationships with disease-specific variables, psychological status, and sleep disturbance. Rheumatol Int 2012;32:2117-24.

28. Zhou W, Guo J, He M, Li J, Chen Y, Liu J, et al. Fatigue and contributing factors in Chinese patients with ankylosing spondylitis. Clin Rheumatol 2020;39:2337-44. 